\title{
A mild and highly chemoselective iodination of alcohol using polymer supported DMAP
}

\author{
DIPARJUN DAS ${ }^{\mathrm{a}}$, JASHA MOMO H ANAL ${ }^{\mathrm{b}}$ and LALTHAZUALA ROKHUM ${ }^{\mathrm{a}, *}$ \\ ${ }^{a}$ Department of Chemistry, National Institute of Technology, Silchar, Assam 788 010, India \\ ${ }^{b}$ Department of Chemistry, North Eastern Hill University, Shillong, Meghalaya 793 022, India \\ e-mail: lalthazualarokhum@gmail.com
}

MS received 9 January 2016; revised 14 July 2016; accepted 13 August 2016

\begin{abstract}
The synthesis of organic compounds using polymer supported catalysts and reagents, where the required product is always in solution, has been of great interest in recent years, both in industries and academia especially in pharmaceutical research. Here, a simple and efficient method for conversion of alcohols into their iodides in high yield using polymer supported 4-(Dimethylamino)pyridine (DMAP) is described. Polymer supported DMAP is used in catalytic amount and is recovered and reused several times. Additionally, this method is highly chemoselective.
\end{abstract}

Keywords. Iodination; Polymer supported DMAP; Triphenylphosphine; Iodine; Catalyst; Chemoselective.

\section{Introduction}

Organo-iodides are widely used in carbon-carbon coupling reactions and as intermediates for organic synthesis in substitution, ${ }^{1}$ elimination and rearrangement reactions. ${ }^{2}$ Presently, organo-iodides are of great use in medical field as well. ${ }^{3,4}$ Many methods for conversion of alcohol to iodide have already been reported in literature ${ }^{5-10}$ but they have a number of drawbacks like high temperature, ${ }^{11}$ long reaction times, ${ }^{12}$ tedious work-up procedures,${ }^{13}$ use of excess reagents, ${ }^{14}$ low yields, drastic conditions, and presence of hazardous vapours. ${ }^{15}$

Development of polymer supported catalysts for organic transformation is one of the important features in organic synthesis because it is reusable and easily separated from the reaction system. ${ }^{16}$ In recent years, the environmental concerns associated with their toxicity, disposal and catalyst/product separation are limiting the applications of homogeneous catalyst in organic syntheses. Therefore, polymer supported catalysts are becoming increasingly important in organic synthesis, primarily due to their low moisture susceptibility, easy handling, minimum side reactions, easy recyclability and reusability. Unlike commonly used acid-base catalysts, polymer supported catalysts offer easy recovery of the catalyst by simple filtration, thereby reducing operational cost.

*For correspondence

\section{Experimental}

\subsection{Materials and Methods}

All the chemicals were used without further purification. All alcohols were of analytical grade. Polymer supported DMAP (4-(Dimethylamino)pyridine, polymer-supported: $\sim 3.0 \mathrm{mmol} / \mathrm{g}$, crosslinked with $2 \%$ DVB) was purchased from Sigma Aldrich and iodine was bought from SpectroChem. Solvents used were extra pure grade purchased from Merck India and were dried by the reported procedure. All compounds were identified by comparing their spectroscopic data with known samples. ${ }^{1} \mathrm{H}$ NMR and ${ }^{13} \mathrm{C}$ NMR spectra of the iodides were performed on Bruker Avance II $400 \mathrm{MHz}$ FT NMR and Bruker Avance III, $500 \mathrm{MHz}$ FT NMR Spectrometer using $\mathrm{CDCl}_{3}$ as solvent.

\subsection{General procedure for the iodination of alcohols}

To a stirred solution of triphenylphosphine $(1.5 \mathrm{mmol})$ in dry dichloromethane was added iodine $(1.5 \mathrm{mmol})$ and polymer supported 4-DMAP (0.4 mmol, $40 \mathrm{~mol} \%)$. Stirring was continued for $2 \mathrm{~min}$; alcohol (1 $\mathrm{mmol})$ was then added. The reaction was monitored by TLC. After complete conversion of the alcohol (as indicated by TLC), the reaction was quenched with an aqueous solution of sodium thiosulfate $(20 \mathrm{~mL})$. The organic solvents were removed and the aqueous solution extracted with ethylacetate $(50 \mathrm{~mL})$. The combined organic layers were dried using sodium sulfate (anhydrous), filtered and 
concentrated. The residue was purified by column chromatography (2\% EtOAc in hexane) to get the desired iodide product.

\subsection{Selected spectral data}

2.3a Benzyl iodide (Table 2, Entry 1): Yellow crystalline solid, M.p.: $22-23^{\circ} \mathrm{C}$, (litt. ${ }^{9,17 h}$ M.p. $21-23^{\circ} \mathrm{C}$ ), ${ }^{1} \mathrm{H}-\mathrm{NMR}\left(500 \mathrm{MHz}, \mathrm{CDCl}_{3}, \mathrm{TMS}\right): \delta 7.34(\mathrm{~d}, J=4.8$ $\mathrm{Hz}, 2 \mathrm{H}), 7.27(\mathrm{t}, J=4.8 \mathrm{~Hz}, 2 \mathrm{H}), 7.20(\mathrm{t}, J=2.68 \mathrm{~Hz}$, $1 \mathrm{H}), 4.42$ (s, 2H); ${ }^{13} \mathrm{C}-\mathrm{NMR}\left(100 \mathrm{MHz}, \mathrm{CDCl}_{3}, \mathrm{TMS}\right)$ : $\delta$ 139.34, 128.89, 128.82, 127.95, 77.47, 76.04, 5.94; IR (KBr): v 2917, 1589, 1147, 1050, 830, $639 \mathrm{~cm}^{-1}$; HRMS: $\mathrm{m} / \mathrm{z} 217.9590\left(\mathrm{M}^{+}\right)$; Elemental analysis for $\mathrm{C}_{7} \mathrm{H}_{7} \mathrm{I}$ : Calculated (\%); C 38.56, H 3.24; Found(\%): C 38.55, H 3.25.

$2.3 \mathrm{~b}$ 4-Methyl benzyl iodide (Table 2, Entry 2): Pale yellow liquid, (litt. ${ }^{17 \mathrm{~h}}$ ), ${ }^{1} \mathrm{H}-\mathrm{NMR}$ (400 $\mathrm{MHz}, \mathrm{CDCl}_{3}$, TMS): $\delta 7.85$ (d, $J=8 \mathrm{~Hz}, 2 \mathrm{H}), 6.76(\mathrm{~d}, J=8 \mathrm{~Hz}, 2 \mathrm{H})$, 4.14 (s, 2H), $2.40(\mathrm{~S}, 3 \mathrm{H}) ;{ }^{13} \mathrm{C}-\mathrm{NMR}\left(100 \mathrm{MHz}, \mathrm{CDCl}_{3}\right.$, TMS): $\delta$ 137.81, 136.28, 129.54, 128.63, 21.25, 6.22; IR (KBr): v 3020, 2920, 1590, 1455, 1148, 1049, 832, $641 \mathrm{~cm}^{-1}$; HRMS: m/z $231.9748\left(\mathrm{M}^{+}\right)$; Elemental analysis for $\mathrm{C}_{8} \mathrm{H}_{9} \mathrm{I}$ : Calculated (\%): C 41.41, H 3.91; Found (\%): C 41.43, H 3.89.

2.3c 4-Chlorobenzyl iodide (Table 2, Entry 3): Colorless crystalline solid, M.p.: $58-59^{\circ} \mathrm{C}$ (litt. ${ }^{9,17 \mathrm{~h}}$ M.p. 58-60 $\left.{ }^{\circ} \mathrm{C}\right),{ }^{1} \mathrm{H}-\mathrm{NMR}\left(500 \mathrm{MHz}, \mathrm{CDCl}_{3}, \mathrm{TMS}\right): \delta 7.92$ $(\mathrm{d}, J=8 \mathrm{~Hz}, 2 \mathrm{H}), 7.22(\mathrm{~d}, J=8.4 \mathrm{~Hz}, 2 \mathrm{H}), 4.29(\mathrm{~s}, 2 \mathrm{H})$; ${ }^{13} \mathrm{C}-\mathrm{NMR}\left(125 \mathrm{~Hz}, \mathrm{CDCl}_{3}\right.$, TMS): $\delta$ 137.87, 133.62, 129.48, 128.29, 4.19; IR (KBr): v 3010, 2915, 1605, 1505, 1490, 1410, 1205, 1150, 1080, 825, $665 \mathrm{~cm}^{-1}$; HRMS: $\mathrm{m} / \mathrm{z} 251.9203\left(\mathrm{M}^{+}\right)$; Elemental analysis for $\mathrm{C}_{7} \mathrm{H}_{6} \mathrm{ClI}$ : Calculated (\%): C 33.30, H 2.41; Found (\%): C 33.28, H 2.39.

2.3d 4-Bromobenzyl iodide (Table 2, Entry 5): Crystalline white solid, M.p.: $58-59^{\circ} \mathrm{C}$ (litt. ${ }^{11,17 \mathrm{~h}}$ M.p. 57$59^{\circ} \mathrm{C}$ ), ${ }^{1} \mathrm{H}-\mathrm{NMR}\left(500 \mathrm{MHz}, \mathrm{CDCl}_{3}, \mathrm{TMS}\right): \delta 7.43$ (d, $J=3.5 \mathrm{~Hz}, 2 \mathrm{H}), 7.10(\mathrm{~d}, J=4.5 \mathrm{~Hz}, 2 \mathrm{H}), 4.41(\mathrm{~s}, 2 \mathrm{H})$; ${ }^{13} \mathrm{C}-\mathrm{NMR}\left(125 \mathrm{~Hz}, \mathrm{CDCl}_{3}\right.$, TMS): $\delta 138.39,132.01$, 130.42, 121.78, 4.46; IR (KBr): v 3015, 2893, 1600, 1510, 1475, 1398, 1212, 1131, 1067, 776, 651, 532, $469 \mathrm{~cm}^{-1}$; HRMS: m/z $295.8693\left(\mathrm{M}^{+}\right)$; Elemental analysis for $\mathrm{C}_{7} \mathrm{H}_{6} \mathrm{BrI}$ : Calculated (\%); C 28.31, H 2.04; Found (\%): C 28.31, H 2.06.

2.3e 4-Methoxybenzyl iodide (Table 2, Entry 9): Pale yellow solid, M.p.: $25-26^{\circ}$ C, (litt. ${ }^{9} 17 \mathrm{~h}$ M.p. $25-$ $27^{\circ} \mathrm{C}$ ), ${ }^{1} \mathrm{H}-\mathrm{NMR}\left(400 \mathrm{MHz}, \mathrm{CDCl}_{3}\right.$, TMS): $\delta 7.20$ $(\mathrm{d}, J=6.8 \mathrm{~Hz}, 2 \mathrm{H}), 7.10(\mathrm{~d}, J=7.2 \mathrm{~Hz}, 2 \mathrm{H}), 4.06$ (s,2H), $3.76(\mathrm{~s}, 3 \mathrm{H}) ;{ }^{13} \mathrm{C}-\mathrm{NMR}\left(100 \mathrm{MHz}, \mathrm{CDCl}_{3}\right.$, TMS): $\delta$ 153.90, 129.45, 128.30, 114.00, 56.84, 10.92; IR (KBr): v 3022, 2915, 1591, 1478, 1284, 1221, 1127 , $811,710 \mathrm{~cm}^{-1}$; HRMS: m/z $247.9693\left(\mathrm{M}^{+}\right)$; Elemental analysis for $\mathrm{C}_{8} \mathrm{H}_{9} \mathrm{IO}$ : Calculated (\%); C 38.74, H 3.66; Found (\%): C 38.76, H 3.64.

$2.3 \mathrm{f}$ (2-Iodoethyl)benzene (Table 2, Entry 10): Yellow liquid, (litt. ${ }^{17 \mathrm{~h}}$ ), ${ }^{1} \mathrm{H}-\mathrm{NMR}\left(400 \mathrm{MHz}, \mathrm{CDCl}_{3}\right.$, TMS): $\delta 7.22(\mathrm{~m}, 3 \mathrm{H}), 7.16(\mathrm{t}, J=6.8 \mathrm{~Hz}, 2 \mathrm{H}), 3.30$ $(\mathrm{t}, J=8 \mathrm{~Hz}, 2 \mathrm{H}), 3.13(\mathrm{t}, J=7.6 \mathrm{~Hz}, 2 \mathrm{H}) .{ }^{13} \mathrm{C}-\mathrm{NMR}$ (100 MHz, $\mathrm{CDCl}_{3}$, TMS): $\delta 140.67,128.49,128.08$, 127.14, 126.92, 126.59, 40.40, 5.80; IR (KBr): v 3029, 2975, 2856, 1904, 1571, 1503, 1483, 1198, 1137, 832, $691 \mathrm{~cm}^{-1}$; HRMS: m/z $231.9746\left(\mathrm{M}^{+}\right)$; Elemental analysis for $\mathrm{C}_{8} \mathrm{H}_{9} \mathrm{I}$ : Calculated (\%); C 41.41, H 3.91; Found (\%): C 41.42, H 3.90.

2.3g 1-Iodooctane (Table 2, Entry 15): Colorless liquid, (litt. ${ }^{17 \mathrm{~h}}$ ), ${ }^{1} \mathrm{H}-\mathrm{NMR}$ (400 MHz, $\mathrm{CDCl}_{3}$, TMS): $\delta$ $3.17(\mathrm{t}, J=6.8 \mathrm{~Hz}, 2 \mathrm{H}), 1.78(\mathrm{~m}, 2 \mathrm{H}), 1.36(\mathrm{t}, J=6.4$ $\mathrm{Hz}, 2 \mathrm{H}), 1.27$ (s, 4H), 0.865 (t, $J=6.8 \mathrm{~Hz}, 3 \mathrm{H}) .{ }^{13} \mathrm{C}-$ NMR (100 MHz, $\mathrm{CDCl}_{3}$, TMS): $\delta 33.57,30.52,29.71$, 29.10, 28.52, 22.64, 14.10, 7.39; IR (KBr): v 2421, 1822, 1573, 1454, 1214, 944, 913, 863, 712, $452 \mathrm{~cm}^{-1}$; HRMS: $\mathrm{m} / \mathrm{z} 240.0376\left(\mathrm{M}^{+}\right)$; Elemental analysis for $\mathrm{C}_{8} \mathrm{H}_{17} \mathrm{I}$ : Calculated (\%); C 40.02, H 7.14; Found (\%): C 40.01, H 7.15.

2.3h 1-Iodododecane (Table 2, Entry 16): Colorless liquid, (litt. ${ }^{17 \mathrm{~h}}$ ), ${ }^{1} \mathrm{H}-\mathrm{NMR}\left(500 \mathrm{MHz}, \mathrm{CDCl}_{3}, \mathrm{TMS}\right): \delta$ $3.18(\mathrm{t}, J=10 \mathrm{~Hz}, 2 \mathrm{H}), 1.81(\mathrm{q}, J=10 \mathrm{~Hz}, 2 \mathrm{H}), 1.55$ $(\mathrm{s}, 2 \mathrm{H}), 1.38(\mathrm{~m}, J=5 \mathrm{~Hz}, 10 \mathrm{H}), 0.87(\mathrm{t}, J=5 \mathrm{~Hz}$, $3 \mathrm{H}) ;{ }^{13} \mathrm{C}-\mathrm{NMR}\left(125 \mathrm{~Hz}, \mathrm{CDCl}_{3}\right.$, TMS $): \delta 33.59,31.91$, $30.52,29.70,29.62,29.55,29.43,29.34,28.55,22.69$, 14.13, 7.36; IR (KBr): v 2422, 1812, 1577, 1455, 1217 , 947, 915, 865, 710, $458 \mathrm{~cm}^{-1}$; HRMS: m/z 296.1003 $\left(\mathrm{M}^{+}\right)$; Elemental analysis for $\mathrm{C}_{12} \mathrm{H}_{25} \mathrm{I}$ : Calculated (\%); C 48.65, H 8.51; Found (\%): C 48.64, H 8.49.

2.3i 1-Iodooctadecane (Table 2, Entry 17): White crystalline solid, M.p.: $34-35^{\circ} \mathrm{C},{ }^{1} \mathrm{H}-\mathrm{NMR}$ (400 MHz, $\left.\mathrm{CDCl}_{3}, \mathrm{TMS}\right): \delta 3.09(\mathrm{t}, J=7.04 \mathrm{~Hz}, 2 \mathrm{H}), 1.748$ $(\mathrm{m}, 2 \mathrm{H}), 1.31(\mathrm{~m}, 2 \mathrm{H}), 1.19(\mathrm{~m}, 24 \mathrm{H}), 0.81(\mathrm{t}, J=$ $6.6 \mathrm{~Hz}, 3 \mathrm{H}) ;{ }^{13} \mathrm{C}-\mathrm{NMR}\left(100 \mathrm{MHz}, \mathrm{CDCl}_{3}, \mathrm{TMS}\right): \delta$ 33.66, 32.01, 30.61, 29.65, 29.46, 28.65, 22.77, 14.20, 7.01; IR (KBr): v 2912, 2817, 1438, 1376, 983, 846, 752, $683 \mathrm{~cm}^{-1}$; HRMS: m/z $380.1938\left(\mathrm{M}^{+}\right)$; Elemental analysis for $\mathrm{C}_{18} \mathrm{H}_{37} \mathrm{I}$ : Calculated (\%); C 56.83, H 9.80; Found (\%): C 56.84, H 9.79. 
2.3j Citronellyl iodide (Table 2, Entry 19): Colorless liquid, (litt. $\left.{ }^{17 \mathrm{~h}}\right),{ }^{1} \mathrm{H}-\mathrm{NMR}\left(400 \mathrm{MHz}, \mathrm{CDCl}_{3}, \mathrm{TMS}\right)$ : $\delta 5.07(\mathrm{t}, J=6.8 \mathrm{~Hz}, 1 \mathrm{H}), 3.21(\mathrm{~m}, 2 \mathrm{H}), 1.85(\mathrm{~m}$, $2 \mathrm{H}), 1.68(\mathrm{~s}, 3 \mathrm{H}), 1.60(\mathrm{~s}, 3 \mathrm{H}), 1.53(\mathrm{~m}, 2 \mathrm{H}), 1.29$ $(\mathrm{m}, 2 \mathrm{H}), 1.14(\mathrm{~m}, 1 \mathrm{H}), 0.87(\mathrm{~d}, J=6.4 \mathrm{~Hz}, 3 \mathrm{H})$; ${ }^{13} \mathrm{C}-\mathrm{NMR}\left(100 \mathrm{MHz}, \mathrm{CDCl}_{3}\right.$, TMS): $\delta 131.45,124.47$, 40.92, 36.33, 33.58, 25.75, 25.33, 18.67, 17.71, 5.14; IR (KBr): v 2931, 2411, 1513, 1423, 1353, 1278, 994, 766, $701 \mathrm{~cm}^{-1}$; HRMS: m/z $266.0530\left(\mathrm{M}^{+}\right)$; Elemental analysis for $\mathrm{C}_{10} \mathrm{H}_{19} \mathrm{I}$ : Calculated (\%); C 45.13, H 7.20; Found (\%): C 45.14, H 7.19.

2.3k Iodocyclohexane (Table 2, Entry 21): Light yellow semi liquid, (litt. ${ }^{17 \mathrm{~h}}$ ), ${ }^{1} \mathrm{H}-\mathrm{NMR}$ (400 $\mathrm{MHz}$, $\left.\mathrm{CDCl}_{3}, \mathrm{TMS}\right): \delta 4.27(\mathrm{~m}, 1 \mathrm{H}), 2.15(\mathrm{~d}, J=9.6 \mathrm{~Hz}, 2 \mathrm{H})$ $2.00(\mathrm{~d}, J=14.4 \mathrm{~Hz}, 2 \mathrm{H}), 1.92(\mathrm{t}, J=12.8 \mathrm{~Hz}, 2 \mathrm{H})$, $1.60(\mathrm{t}, J=20 \mathrm{~Hz}, 2 \mathrm{H}), 1.39(\mathrm{~d}, J=8 \mathrm{~Hz}, 2 \mathrm{H}) ;{ }^{13} \mathrm{C}-$ NMR (100 MHz, $\mathrm{CDCl}_{3}$, TMS): $\delta$ 39.60, 32.85, 27.32, 25.20; IR (KBr): v 2419, 1820, 1575, 1455, 1224, 954, 916, 865, 715, $455 \mathrm{~cm}^{-1}$; HRMS: m/z $209.9907\left(\mathrm{M}^{+}\right)$; Elemental analysis for $\mathrm{C}_{6} \mathrm{H}_{11} \mathrm{I}$ : Calculated (\%); C 34.31, H 5.28; Found (\%): C 34.33, H 5.26.

2.31 1-Bromo-4-(2-bromoethyl)benzene (Table 2, Entry 28): White crystalline solid, M.p.: $95-96^{\circ} \mathrm{C}$,

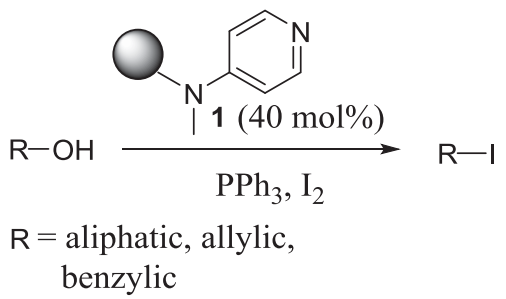

Scheme 1. Iodination of alcohols.
(400 MHz, $\left.\mathrm{CDCl}_{3}, \mathrm{TMS}\right): \delta 7.92(\mathrm{~d}, J=7.6 \mathrm{~Hz}, 2 \mathrm{H})$, $7.21(\mathrm{~d}, J=7.6 \mathrm{~Hz}, 2 \mathrm{H}), 3.82(\mathrm{t}, J=7.2 \mathrm{~Hz}, 2 \mathrm{H})$, 3.06 (t, $J=7.2 \mathrm{~Hz}, 2 \mathrm{H}) ;{ }^{13} \mathrm{C}-\mathrm{NMR}\left(100 \mathrm{MHz}, \mathrm{CDCl}_{3}\right.$, TMS): $\delta$ 143.40, 132.09, 132.00, 115.34, 40.36, 31.91; HRMS: $\mathrm{m} / \mathrm{z} 261.8990\left(\mathrm{M}^{+}\right)$; Elemental analysis for $\mathrm{C}_{8} \mathrm{H}_{8} \mathrm{Br}_{2}$ : Calculated (\%); C 36.40, H 3.06; Found (\%): C 36.42, H 3.04.

\section{Results and Discussion}

In continuation of our on-going interest for the development of useful synthetic methodologies,${ }^{17}$ herein, we have reported the synthesis of organo-halide compounds using catalytic amount of polymer supported DMAP, 1. Iodination of alcohols to its corresponding iodides was achieved using triphenylphosphine and iodine reagent system with excess base which is assumed to neutralize the hydroiodic acid generated in the reaction. ${ }^{12,14}$ But Hajipour and co-workers ${ }^{11}$ have shown that the conversion could be achieved without the reaction system being neutral. This finding prompted us to investigate the iodination reaction using catalytic amount of base (Scheme 1).

To achieve this, iodination of benzyl alcohol was carried out at room temperature by changing different parameters as given in Table 1. Among the various solvents tried, dichloromethane was found to be the best solvent for the transformation (Table 1, entry 4). After optimising the solvents, our next obvious target

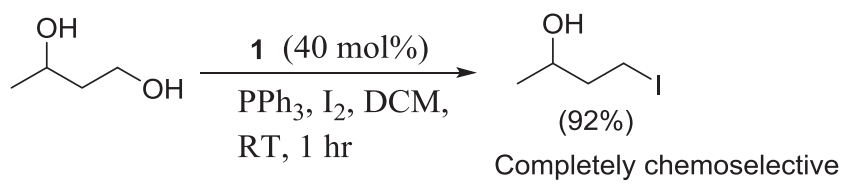

Scheme 2. Chemoselective iodination.

Table 1. Optimization of reaction conditions $\mathrm{s}^{\mathrm{a}}$.

\begin{tabular}{|c|c|c|c|c|}
\hline Entry & Mol $\%$ of catalyst 1 used & Solvent & Time (h) & Conversion $(\%)$ \\
\hline 1. & 40 & $\mathrm{CH}_{3} \mathrm{CN}$ & 2 & 51 \\
\hline 2. & 10 & $\mathrm{CH}_{2} \mathrm{Cl}_{2}$ & 3 & 31 \\
\hline 3. & 20 & $\mathrm{CH}_{2} \mathrm{Cl}_{2}$ & 2 & 82 \\
\hline 4. & 40 & $\mathrm{CH}_{2} \mathrm{Cl}_{2}$ & 1 & Quantitative \\
\hline 5. & - & $\mathrm{CH}_{2} \mathrm{Cl}_{2}$ & 3 & 12 \\
\hline 6. & 50 & EtOAc & 2 & 61 \\
\hline 7. & 40 & $\mathrm{CHCl}_{3}$ & 2 & 84 \\
\hline 8. & 40 & THF & 3 & 71 \\
\hline 9. & 50 & Toluene & 3 & 45 \\
\hline
\end{tabular}

${ }^{a}$ Reaction condition: alcohol $(1 \mathrm{mmol}), \mathrm{PPh}_{3}(1.5 \mathrm{mmol})$, iodine $(1.5 \mathrm{mmol})$, solvent, room temperature. 
Table 2. Iodination of alcohol with polymer supported DMAP ${ }^{a}$.

\begin{tabular}{|c|c|c|c|c|}
\hline Entry & Alcohol & Product & Time(h) & $\%$ Yield $^{b}$ \\
\hline 1. & & & 1 & 95 \\
\hline 2. & & & 1 & 92 \\
\hline 3. & & & 1.5 & 94 \\
\hline 4. & & & 2 & 89 \\
\hline 5. & & & 1.5 & 90 \\
\hline 6. & & & 1.5 & 87 \\
\hline 7. & & & 2 & 96 \\
\hline 8. & & & 2 & 91 \\
\hline 9. & & & 1 & 97 \\
\hline 10. & & & 1.5 & 88 \\
\hline 11. & & & 1.5 & 90 \\
\hline 12. & & & 2 & 92 \\
\hline 13. & & & 3 & 87 \\
\hline 14. & & & 1 & 92 \\
\hline 15. & & & 1 & 95 \\
\hline
\end{tabular}


Table 2. (continued)

\begin{tabular}{|c|c|c|c|c|}
\hline Entry & Alcohol & Product & Time(h) & $\%$ Yield $^{b}$ \\
\hline 16. & & & 1.5 & 89 \\
\hline 17. & & & 1.5 & 86 \\
\hline 18. & & & 1.2 & 90 \\
\hline 19. & & & 1 & 95 \\
\hline 20. & & & 1 & 90 \\
\hline 21. & & & 3 & 89 \\
\hline 22. & & & 3 & 0 \\
\hline 23 & & & 12 & 84 \\
\hline 24. & & & 3 & 87 \\
\hline 25. & & & 3.5 & 84 \\
\hline 26. & & & 4 & 89 \\
\hline 27. & & & 2 & $91^{c}$ \\
\hline 28. & & & 2 & $90^{c}$ \\
\hline
\end{tabular}

${ }^{a}$ Reaction condition: alcohol $(1 \mathrm{mmol}), \mathrm{PPh}_{3}(1.5 \mathrm{mmol})$, iodine $(1.5 \mathrm{mmol})$ DMAP $(0.4 \mathrm{mmol}, 40 \mathrm{~mol} \%), \mathrm{DCM}, \mathrm{room}$ temperature. ${ }^{b}$ Isolated yield. ${ }^{c}$ Bromine $\left(\mathrm{Br}_{2}\right)$ were used in place of iodine. 
Table 3. Chemoselective iodination of alcohol with polymer supported DMAP ${ }^{a}$.

Entry

${ }^{a}$ Reaction condition: alcohol $(1 \mathrm{mmol}), \mathrm{PPh}_{3}(1.5 \mathrm{mmol})$, iodine $(1.5 \mathrm{mmol})$, DMAP $(0.4 \mathrm{mmol}, 40 \mathrm{~mol} \%)$, DCM, room temperature. ${ }^{b}$ Isolated yield. ${ }^{c}$ Bromine was used in place of iodine.

is to optimize the amount of base catalyst and reagents required. At the outset, different quantities of catalyst and reagents were investigated and we found that use of $40 \mathrm{~mol} \%$ DMAP, 1 with molar ratio of 1.5:1.5:1 for triphenylphosphine/iodine/alcohol gave optimum yield.

With the optimized reaction condition in our hand, we examined a variety of primary, secondary, tertiary and benzylic alcohols carrying electron-donating and electron withdrawing groups to determine the scope of this procedure. The results are given in Table 2. The results for the iodination under our optimized conditions were, in general, good to excellent. Thus, when benzyl alcohol (Table 2, entry 1) and short aliphatic alcohols were used (Table 2, entries 14-15), the corresponding iodides were obtained in excellent yield. However, when longer aliphatic chains were used, lower yields were observed (Table 2, entries 16-18). It was observed that, substrates with electron withdrawing group in the benzene ring took maximum time (Table 2, entries 7-8) whereas electron-donating substituent group reduced the time for complete consumption of the starting alcohol (Table 2, entry 9). Secondary alcohols required longer reaction time for complete conversion compared to their primary counterparts (Table 2, entry 1 vs. 12). However, the conversion of phenol into its corresponding iodide was not successful even after prolonged stirring (Table 2 , entry 22). To our pleasure, tertiary alcohols were also successfully converted to their corresponding iodides (Table 2, entries 25-26). After exploring the iodination of varieties of alcohols, we turned our attention towards the possibility of direct bromination using bromine $\left(\mathrm{Br}_{2}\right)$ under our optimized reaction condition used for iodination. Gratifyingly, we found that the bromination of alcohols also occurred equally well giving the desired bromides in high yields (Table 2, entries 27-28).

Chemoselective iodination of unsymmetrical diols was also carried out under our optimized reaction conditions (Scheme 2). Our system works well for the selective iodination of a variety of unsymmetrical diols in favour of the less hindered alcohols (Table 3). It has been observed that primary alcohol moiety solely undergoes iodination discriminating the secondary alcohol. Hence, butane-1,3-diol exclusively gave 4iodobutan-2-ol in $92 \%$ yield (Table 3, entry 1 ). Chemoselective iodination of 2-methylpentane-2,4-diol to yield 4-iodo-2-methylpentan-2-ol was also successfully carried out showing complete selectivity of our protocol (Table 3, entry 7).

Recovery and reusability of the catalyst are very important factors for practical application of the heterogeneous system. The reusability of the catalyst was 
also investigated using benzyl alcohol as a test substrate using our optimized reaction conditions. After completion of the first set of reaction (run 1) to afford the corresponding product in $95 \%$ yield within 1 hour, the catalyst was recovered by simply washing with ethyl acetate and drying it at $100^{\circ} \mathrm{C}$ in Abderhalden apparatus under reduced pressure. A new reaction was then performed with fresh solvent and reactants under the same condition. To our satisfaction, catalyst could be reused at least four times without any noticeable change in activity (run 2, 3 and 4 gave 95\%, 93\% and 92\% yield, respectively).

\section{Conclusions}

In conclusion, we have developed an efficient and highly chemoselective method of iodination under very mild condition by using triphenylphosphine, iodine and catalytic amount of polymer supported 4-DMAP. The catalyst could be regenerated very easily and reused several times without loss in efficiency, thus making the reaction cost effective and environmentally benign.

\section{Supporting information (SI)}

Spectral data $\left({ }^{1} \mathrm{H}\right.$ NMR and ${ }^{13} \mathrm{C}$ NMR) are available as Supplementary Information at www.ias.ac.in/chemsci.

\section{Acknowledgements}

We gratefully acknowledge Science and Engineering Research Board (SERB)-DST, Govt. of India, New Delhi for financial supports [sanctioned no: SB/FT/CS103/2013, SB/EMEQ-076/2014].

\section{References}

1. (a) Frisch A C and Beller M 2005 Angew. Chem. Int. Ed. 44 674; (b) Su M D and Chu S Y 1999 J. Am. Chem. Soc. 1211045

2. Naso F and Marchese G 1983 In Carbon-carbon bond formation involving organic halides and transition metal compounds. The Chemistry halides, pseudo-halides and azides S Patai and Z J Rappoport (Eds.) (New York: Wiley) p.1353

3. Abraham G E and Flechas J E 2007 The Original Internist 158

4. Arroyo-Helguera O, Rojas E, Delgado G and Aceves C 2008 Endocr. Relat. Cancer 151003

5. Hajipour A R, Mostafavi M and Ruoho A E 2009 Org. Prep. Proced. Int. 4187

6. Jung M E and Ornstein P L 1977 Tetrahedron. Lett. 31 2659

7. Martinez A G, Alvarez R M, Vilar E T, Fraile A G, Barnica J O, Hanack M and Subramanian L R 1987 Tetrahedron Lett. 286441

8. Firouzabadi H, Iranpoor N and Jafarpour M 2004 Tetrahedron Lett. 457451

9. Hayat S, Atta-ur-Rahman, Khan K M, Choudhary M I, Maharvi G M, Zia-Ullah and Bayer E 2003 Synth. Commun. 332531

10. Firouzabadi H, Iranpoor N and Ebrahimzadeh F 2006 Tetrahedron Lett. 471171

11. Hajipour A R, Falahati A R and Ruoho A E 2006 Tetrahedron Lett. 474191

12. (a) Garegg P J and Samuelsson G J 1979 J. Chem. Soc., Chem. Commun. 978; (b) Garegg P J, Johansson R, Ortega C and Samuelsson G J 1982 Chem. Soc., Perkin Trans. 1681

13. Tundo P and Venturello P 1979 Synthesis 12 952

14. (a) Anilkumar G, Nambu H and Kita Y 2002 Org. Proc. Res. Dev. 6 190; (b) Rokhum L, Vanlalveni C and Lalfakzuala R 2013 IJETCAS 13370

15. Appel R 1975 Angew. Chem. Int. Ed. Engl. 14801

16. (a) Hodge P 2005 Ind. Eng. Chem. Res. 44 8542; (b) Gladysz J A 2002 Chem. Rev. 103 3216; (c) McNamara C A, Dixon M J and Bradley M 2002 Chem. Rev. 102 3275; (d) Sandaroos R, Goldani M T, Damavandi S and Mohammadi A 2012 J. Chem. Sci. 124 871; (e) Islam N S and Boruah J J 2015 J. Chem. Sci. 127777

17. (a) Pathak G and Rokhum L 2015 ACS Comb. Sci. 17 483; (b) Rokhum L and Bez 2013 Can. J. Chem. 91 300; (c) Rokhum L and Bez G 2013 Tetrahedron Lett. 54 5500; (d) Rokhum L and Bez G 2011 J. Chem. Sci. 124 687; (e) Bora P P, Vanlaldinpuia K, Rokhum L and Bez G 2011 Synth. Commun. 41 2674; (f) Rokhum L and Bez G 2011 Synth. Commun. 41 548; (g) Vanlaldinpuia K, Sema H A, Rokhum L and Bez G 2010 Chem. Lett. 39 228; (h) Das D, Chanda T and Rokhum L 2015 Acta Chim. Slov. 62775 